\title{
SECOND METHOD OF LYAPUNOV AND COMPARISON PRINCIPLE FOR IMPULSIVE DIFFERENTIAL-DIFFERENCE EQUATIONS
}

\author{
D. D. BAINOV ${ }^{1}$ and I. M. STAMOVA ${ }^{2}$
}

(Received 29 March 1995; revised 18 September 1995)

\begin{abstract}
In the present paper questions related to stability and boundedness with respect to manifolds of solutions of impulsive differential-difference equations are considered. The investigations are carried out by means of piecewise-continuous functions which are analogues of the classical Lyapunov's functions. By means of a vectorial comparison equation and differential inequalities for piecewise-continuous functions, theorems are proved on stability and boundedness with respect to manifolds of solutions of impulsive differential-difference equations with impulse effect at fixed moments.
\end{abstract}

\section{Introduction}

A number of processes studied in physics, chemistry, biology, etc., are characterized by the fact that at fixed moments they change their state by jumps. Adequate mathematical models of such processes are the impulsive differential equations.

The beginning of the investigations devoted to the impulsive differential equations was set with the work of Mil'man and Myshkis [6]. In the recent years the theory of these equations is developing very intensively due to their numerous applications to radio engineering, control theory, biotechnologies, industrial robotics, economics, etc. (Bainov and Simeonov [2], Kulev and Bainov [4], Lakshmikantham, Bainov and Simeonov [5], Simeonov and Bainov [7], Vasundhara Devi [8].)

The impulsive differential-difference equations are a generalization of the impulsive differential equations. They are mathematical models of real processes which, besides the change by jumps of the state, are characterized by a dependence on their prehistory. In spite of the great possibilities for application, the theory of the impulsive differentialdifference equations is developing rather slowly (Bainov, Covachev and Stamova [1]).

\footnotetext{
${ }^{1}$ Medical University, P.O. Box 45, Sofia 1504, Bulgaria

${ }^{2}$ Technical University, Sliven, Bulgaria

(C) Australian Mathematical Society, 1997, Serial-fee code 0334-2700/97
} 
In the present paper questions related to stability and boundedness with respect to manifolds of solutions of an impulsive system of differential-difference equations with impulse effect at fixed moments are considered. The investigations are carried out by means of piecewise continuous functions which are analogues of the classical Lyapunov's functions (Simeonov and Bainov [7]).

\section{Preliminary notes and definitions}

Denote by $\mathbb{R}^{n}$ the $n$-dimensional Euclidean space with elements $x=$ $\operatorname{col}\left(x_{1}, \ldots, x_{n}\right)$ and the norm $|x|=\left(\sum_{i=1}^{n} x_{i}^{2}\right)^{1 / 2}, h>0, \varphi_{0}:\left[t_{0}-h, t_{0}\right] \rightarrow \mathbb{R}^{n}$. Consider an impulsive system of differential-difference equations of the form

$$
\begin{aligned}
\dot{x}(t) & =f(t, x(t), x(t-h)), \quad t \neq \tau_{i}, \quad t>t_{0}, \\
x(t) & =\varphi_{0}(t), \quad t \in\left[t_{0}-h, t_{0}\right], \\
\Delta x\left(\tau_{i}\right) & =x\left(\tau_{i}+0\right)-x\left(\tau_{i}-0\right)=I_{i}\left(x\left(\tau_{i}\right)\right), \quad \tau_{i}>t_{0}, \quad i=1,2, \ldots,
\end{aligned}
$$

where $t_{0} \in \mathbb{R}, f:\left(t_{0}, \infty\right) \times \mathbb{R}^{n} \times \mathbb{R}^{n} \rightarrow \mathbb{R}^{n}, I_{i}: \mathbb{R}^{n} \rightarrow \mathbb{R}^{n}, i=1,2, \ldots$,

$$
t_{0} \equiv \tau_{0}<\tau_{1}<\ldots<\tau_{i}<\tau_{i+1}<\ldots
$$

Denote by $x(t)=x\left(t ; t_{0}, \varphi_{0}\right)$ the solution of the problem (1), and by $J^{+}\left(t_{0}, \varphi_{0}\right)$ the maximal interval of the type $\left[t_{0}, \omega\right)$ in which this solution is defined. Let $\varphi_{0} \in$ $C\left[\left[t_{0}-h, t_{0}\right], \mathbb{R}^{n}\right]$. The solution $x(t)=x\left(t ; t_{0}, \varphi_{0}\right)$ of the problem $(1)$ is characterized by the following:

1. For $t_{0}-h \leq t \leq t_{0}$ the solution $x(t)$ coincides with the function $\varphi_{0}$.

2. The solution $x\left(t ; t_{0}, \varphi_{0}\right)$ is a piecewise-continuous function on $J^{+}\left(t_{0}, \varphi_{0}\right)$ with points of discontinuity of the first kind $\tau_{i}, i=1,2, \ldots$, at which it is continuous from the left, that is, at the moments of the impulse effect $\tau_{i}$ the following equalities are valid:

$$
\begin{aligned}
& x\left(\tau_{i}-0\right)=x\left(\tau_{i}\right), \\
& x\left(\tau_{i}+0\right)=x\left(\tau_{i}\right)+I_{i}\left(x\left(\tau_{i}\right)\right), \quad \tau_{i} \neq \tau_{l}+h, l=0,1,2, \ldots .
\end{aligned}
$$

3. If for some $l=0,1,2 \ldots$ we have $\tau_{i}<\tau_{l}+h<\tau_{i+1}, i=1,2, \ldots$, then in the interval $\left[\tau_{l}+h, \tau_{i+1}\right]$ the solution $x(t)$ of the problem (1) coincides with the solution of the problem

$$
\begin{aligned}
& \dot{y}(t)=f(t, y(t), x(t-h+0)), \\
& y\left(\tau_{l}+h\right)=x\left(\tau_{l}+h\right) .
\end{aligned}
$$


4. If $\tau_{l}+h \equiv \tau_{i}$ for $l=0,1,2 \ldots, i=1,2, \ldots$, then in the interval $\left[\tau_{l}+h, \tau_{i+1}\right]$ the solution $x(t)$ coincides with the solution of the problem

$$
\begin{aligned}
& \dot{y}(t)=f(t, y(t), x(t-h+0)), \\
& y\left(\tau_{l}+h\right)=x\left(\tau_{l}+h\right)+I_{i}\left(x\left(\tau_{l}+h\right)\right) .
\end{aligned}
$$

5. The function $x(t)$ is piecewise differentiable with respect to $t$ and $\dot{x}(t)=$ $f(t, x(t), x(t-h))$ for $t \in J^{+}\left(t_{0}, \varphi_{0}\right) \backslash\left(\left\{\tau_{i}\right\}_{i=1}^{\infty} \cup\left\{\tau_{l}+h\right\}_{l=0}^{\infty}\right)$.

Introduce the following notation: $\mathbb{R}_{+}=[0, \infty), \mathscr{K}$ is the class of all continuous functions $a: \mathbb{R}_{+} \rightarrow \mathbb{R}_{+}$such that $a(0)=0, C_{0}=C\left[\left[t_{0}-h, t_{0}\right], \mathbb{R}^{n}\right],\left\|\varphi_{0}\right\|=$ $\max _{s \in\left[t_{0}-h, t_{0}\right]}\left|\varphi_{0}(s)\right|$ is the norm of the function $\varphi_{0} \in C_{0}$,

$$
G_{i}=\left\{(t, x) \in\left[t_{0}, \infty\right) \times \mathbb{R}^{n}: \tau_{i-1}<t<\tau_{i}\right\}, \quad i=1,2, \ldots,
$$

$\tilde{x}(t)=x(t-h), t \in\left(t_{0}, \infty\right)$.

Let $g:\left[t_{0}-h, \infty\right) \times \mathbb{R}^{n} \rightarrow \mathbb{R}^{m}(m \leq n)$. For $t \in\left[t_{0}-h, \infty\right)$ define the sets

$$
\begin{aligned}
& M_{t}(n-m)=\left\{x \in \mathbb{R}^{n}: g(t, x)=0\right\}, \\
& M_{t}(n-m)(\alpha)=\left\{x \in \mathbb{R}^{n}:|g(t, x)|<\alpha\right\}, \quad t \in\left(t_{0}, \infty\right), \\
& M_{t_{0}}(n-m)(\bar{\alpha})=\left\{\varphi \in C_{0}:\|g(t, \varphi)\|=\max _{t \in\left[t_{0}-h, t_{0}\right]}|g(t, \varphi(t))| \leq \alpha\right\}, \\
& \alpha=\text { const }>0 .
\end{aligned}
$$

We shall use the following definitions of stability and boundedness (Bhatia and Lakshmikantham [3])

DEFINITION 1. The zero solution $x(t) \equiv 0$ of the problem (1) is said to be:

1.1. Stable with respect to the function $g(t, x)$ if for any $t_{0} \in \mathbb{R}$ and any $\varepsilon>0$, there exists a positive function $\delta=\delta\left(t_{0}, \varepsilon\right)$ which is continuous in $t_{0}$ for any fixed $\varepsilon>0$ and such that if $\varphi_{0} \in M_{t_{0}}(n-m)(\bar{\delta})$ and $t \in J^{+}\left(t_{0}, \varphi_{0}\right)$, then $x\left(t ; t_{0}, \varphi_{0}\right) \in M_{t}(n-m)(\varepsilon)$.

1.2. Uniformly stable with respect to the function $g(t, x)$ if the function $\delta$ in 1.1 does not depend on $t_{0}$.

1.3. Globally equi-attractive with respect to the function $g(t, x)$ if for any $t_{0} \in$ $\mathbb{R}, \alpha>0$ and $\varepsilon>0$ there exists a positive number $T=T\left(t_{0}, \alpha, \varepsilon\right)$ such that if $\varphi_{0} \in M_{t_{0}}(n-m)(\bar{\alpha})$, then $t_{0}+T \in J^{+}\left(t_{0}, \varphi_{0}\right)$, and $x\left(t ; t_{0}, \varphi_{0}\right) \in M_{t}(n-m)(\varepsilon)$ for $t \in\left[t_{0}+T, \infty\right) \cap J^{+}\left(t_{0}, \varphi_{0}\right)$.

1.4. Uniformly globally attractive with respect to the function $g(t, x)$ if the number $T$ of 1.3 does not depend on $t_{0}$.

1.5. Globally equi-asymptotically stable with respect to the function $g(t, x)$ if it is stable and globally equi-attractive with respect to the function $g(t, x)$. 
1.6. Uniformly globally asymptotically stable with respect to the function $g(t, x)$ if it is uniformly stable and uniformly globally attractive with respect to the function $g(t, x)$.

1.7. Unstable with respect to the function $g(t, x)$ if there exists $\varepsilon>0$ and $t_{0} \in \mathbb{R}$ such that for any $\delta>0$ one can choose $\varphi_{0} \in M_{t_{0}}(n-m)(\bar{\delta})$ and $t \in J^{+}\left(t_{0}, \varphi_{0}\right)$, such that $\left|g\left(t, x\left(t ; t_{0}, \varphi_{0}\right)\right)\right| \geq \varepsilon$.

DEFINITION 2. The solutions of the problem (1) are said to be:

2.1. Equi-bounded with respect to the function $g(t, x)$ if for any $t_{0} \in \mathbb{R}$ and any $\alpha>0$ there exists a positive function $\beta=\beta\left(t_{0}, \alpha\right)$ which is continuous in $t_{0}$ for any $\alpha>0$ and such that if $\varphi_{0} \in M_{t_{0}}(n-m)(\bar{\alpha}), t \in J^{+}\left(t_{0}, \varphi_{0}\right)$, then $x\left(t ; t_{0}, \varphi_{0}\right) \in$ $M_{t}(n-m)(\beta)$.

2.2. Uniformly bounded with respect to the function $g(t, x)$ if the function $\beta$ in 2.1 does not depend on $t_{0}$.

2.3. Ultimately bounded with respect to the function $g(t, x)$ for bound $N$ if there exists a number $N>0$ and for any $t_{0} \in \mathbb{B}$ and $\alpha>0$ there exists a positive number $T=T\left(t_{0}, \alpha\right)$ such that if $\varphi_{0} \in M_{t_{0}}(n-m)(\bar{\alpha})$, then $t_{0}+T \in J^{+}\left(t_{0}, \varphi_{0}\right)$ and $x\left(t ; t_{0}, \varphi_{0}\right) \in M_{t}(n-m)(N)$ for $t \in\left[t_{0}+T, \infty\right) \cap J^{+}\left(t_{0}, \varphi_{0}\right)$.

2.4. Uniformly ultimately bounded with respect to the function $g(t, x)$ for bound $N$ if the number $T$ from 2.3 does not depend on $t_{0}$.

REMARK. If $n=m$ and $g(t, x) \equiv x$, then Definition 1 is reduced to the definition of stability by Lyapunov of the zero solution of the problem (1) and Definition 2 is reduced to the definition of boundedness of the solutions of the problem (1).

Together with the problem (1) we shall consider the problem

$$
\begin{aligned}
& \dot{u}(t)=F(t, u(t)), \quad t \neq \tau_{i}, \quad t>t_{0}, \\
& u\left(t_{0}+0\right)=u_{0}, \\
& \Delta u\left(\tau_{i}\right)=B_{i}\left(u\left(\tau_{i}\right)\right), \quad \tau_{i}>t_{0}, \quad i=1,2, \ldots,
\end{aligned}
$$

where $u_{0} \in \Omega, F:\left(t_{0}, \infty\right) \times \Omega \rightarrow \mathbb{R}^{k}, B_{i}: \Omega \rightarrow \mathbb{R}^{k}, i=1,2, \ldots, \Omega$ is a domain in $\mathbb{R}^{k}$ containing the origin.

Denote by $u(t)=u\left(t ; t_{0}, u_{0}\right)$ the solution of the problem (2) and by $J^{+}\left(t_{0}, u_{0}\right)$ the maximal interval of the form $\left[t_{0}, \omega\right)$ in which the solution $u\left(t ; t_{0}, u_{0}\right)$ is defined. Introduce into $\mathbb{R}^{k}$ a partial ordering in the following way: for the vectors $u, v \in \mathbb{R}^{k}$ we shall say that $u \geq v$ if $u_{j} \geq v_{j}$ for each $j=1,2, \ldots, k$ and $u>v$ if $u_{j}>v_{j}$ for each $j=1,2, \ldots, k$.

DEFINITION 3. The function $\psi: \Omega \rightarrow \mathbb{R}^{k}$ is said to be monotone increasing in $\Omega$ if $\psi(u)>\psi(v)$ for $u>v$ and $\psi(u) \geq \psi(v)$ for $u \geq v, u, v \in \Omega$. 
DEFINITION 4. The function $F:\left(t_{0}, \infty\right) \times \Omega \rightarrow \mathbb{R}^{k}$ is said to be quasimonotone increasing in $\left(t_{0}, \infty\right) \times \Omega$ if for any two points $(t, u)$ and $(t, v)$ in $\left(t_{0}, \infty\right) \times \Omega$ and for any $j=1,2, \ldots, k$ the inequality $F_{j}(t, u) \geq F_{j}(t, v)$ is valid only if $u_{j}=v_{j}$ and $u \geq v$, that is, if for any fixed $t \in\left(t_{0}, \infty\right)$ and any $j=1,2, \ldots, k$ the function $F_{j}(t, u)$ is nondecreasing with respect to $\left(u_{1}, \ldots, u_{j-1}, u_{j+1}, \ldots, u_{k}\right)$.

DefinITION 5. The solution $u^{+}: J^{+}\left(t_{0}, u_{0}\right) \rightarrow \mathbb{R}^{k}$ of the problem (2) is said to be a maximal solution if any other solution $u:\left(t_{0}, \tilde{\omega}\right) \rightarrow \mathbb{R}^{k}$ satisfies the inequality $u^{+}(t) \geq u(t)$ for $t \in J^{+}\left(t_{0}, u_{0}\right) \cap\left(t_{0}, \tilde{\omega}\right)$.

A minimal solution $u^{-}(t)$ of the problem (2) is defined in an analogous way.

Let $e \in \mathbb{R}^{k}$ be the vector $(1,1, \ldots, 1)$ and $\{u: 0 \leq u \leq e\} \subset \Omega$. Further on we shall consider only such solutions $u(t)$ of the problem (2) for which $u(t) \geq 0$. That is why the following definitions of stability and boundedness of the solutions of (2) are appropriate:

DEFINITION 6. The zero solution of the problem (2) is said to be:

6.1. Stable if for any $t_{0} \in \mathbb{R}$ and $\varepsilon>0$ there exists a positive function $\delta=\delta\left(t_{0}, \varepsilon\right)$ which is continuous in $t_{0}$ for any $\varepsilon>0$ and is such that if $0 \leq u_{0} \leq \delta e$ and $t \in J^{+}\left(t_{0}, u_{0}\right)$, then $u^{+}\left(t ; t_{0}, u_{0}\right)<\varepsilon e$.

6.2. Uniformly stable if the function $\delta$ in 6.1 does not depend on $t_{0}$.

6.3. Globally equi-attractive if for any $t_{0} \in \mathbb{R}, \alpha>0$ and $\varepsilon>0$ there exists a positive number $T=T\left(t_{0}, \alpha, \varepsilon\right)$ such that if $0 \leq u_{0} \leq \alpha e$, then $t_{0}+T \in J^{+}\left(t_{0}, u_{0}\right)$ and $u^{+}\left(t ; t_{0}, u_{0}\right)<\varepsilon e$ for $t \in\left[t_{0}+T, \infty\right) \cap J^{+}\left(t_{0}, u_{0}\right)$.

6.4. Uniformly globally attractive if the number $T$ of 6.3 does not depend on $t_{0}$.

6.5. Globally equi-asymptotically stable if it is stable and globally equi-attractive.

6.6. Uniformly globally asymptotically stable if it is uniformly stable and uniformly globally attractive.

6.7. Unstable if there exists $\varepsilon>0$ and $t_{0} \in \mathbb{R}$ such that for any $\delta>0$ one can choose $u_{0} \in \Omega, 0 \leq u_{0} \leq \delta e$ and $t>t_{0}$ so that the inequality $u^{-}\left(t ; t_{0}, u_{0}\right) \nless \varepsilon e$ is valid.

(We shall note that the symbol $\Varangle$ is not equivalent to the symbol $\geq$ in general, and it means that there exists $j=1,2, \ldots, k$ such that $u_{j}^{-}\left(t ; t_{0}, u_{0}\right) \geq \varepsilon$.)

DEFINITION 7. The solutions of the problem (2) are said to be:

7.1. Equi-bounded if for any $t_{0} \in \mathbb{R}$ and any $\alpha>0$ there exists a positive function $\beta=\beta\left(t_{0}, \alpha\right)$ which is continuous in $t_{0}$ for any $\alpha>0$ and such that if $0 \leq u_{0} \leq \alpha e$ and $t \in J^{+}\left(t_{0}, u_{0}\right)$, then $u^{+}\left(t ; t_{0}, u_{0}\right) \leq \beta e$.

7.2. Uniformly bounded if the function $\beta$ in 7.1 does not depend on $t_{0}$.

7.3. Ultimately bounded for bound $N$ if there exists a number $N>0$ and for any $t_{0} \in \mathbb{R}$ and $\alpha>0$ there exists a positive number $T=T\left(t_{0}, \alpha\right)$ such that if $0 \leq u_{0} \leq \alpha e$, 
then $t_{0}+T \in J^{+}\left(t_{0}, u_{0}\right)$ and $u^{+}\left(t ; t_{0}, u_{0}\right)<N e$ for $t \in\left[t_{0}+T, \infty\right) \cap J^{+}\left(t_{0}, u_{0}\right)$.

7.4. Uniformly ultimately bounded for bound $N$ if the number $T$ in 7.3 does not depend on $t_{0}$.

Henceforth we shall use piecewise-continuous auxiliary functions which are analogues of the classical Lyapunov's functions (Simeonov and Bainov [7]).

DEFINITION 8. We say that the function $V:\left[t_{0}, \infty\right) \times \mathbb{R}^{n} \rightarrow \Omega, V=\left(V_{1}, \ldots, V_{k}\right)$ belongs to the class $\mathscr{V}_{0}$ if it satisfies the following conditions.

1. The function $V$ is continuous in any of the sets $G_{i}, i=1,2, \ldots$ and $V(t, 0)=0$ for $t \in\left[t_{0}, \infty\right)$.

2. The function $V$ is Lipschitz continuous with respect to its second argument $x$ in any of the sets $G_{i}, i=1,2, \ldots$.

3. For each $i=1,2, \ldots$ there exist the finite limits

$$
V\left(\tau_{i}-0, x\right)=\lim _{\substack{t \rightarrow \tau_{i} \\(t, x) \in G_{i}}} V(t, x), \quad V\left(\tau_{i}+0, x\right)=\lim _{\substack{t \rightarrow \tau_{i} \\(t, x) \in C_{i+1}}} V(t, x)
$$

4. The equality $V\left(\tau_{i}-0, x\right)=V\left(\tau_{i}, x\right), x \in \mathbb{R}^{n}$ is valid.

Let $V \in \mathscr{V}_{0}$. For $(t, x) \in \cup_{i=1}^{\infty} G_{i}$ we define the function

$$
\dot{V}_{(1)}(t, x)=\limsup _{\sigma \rightarrow 0^{+}} \sigma^{-1}[V(t+\sigma, x+\sigma f(t, x, \tilde{x}))-V(t, x)] .
$$

If $x=x(t)$ is the solution of the problem (1), then

$$
\dot{V}_{(1)}(t, x(t))=D^{+} V(t, x(t)), \quad t \neq \tau_{i}, i=1,2, \ldots,
$$

where $D^{+} V(t, x(t))$ is the right Dini derivative of the function $V(t, x(t))$.

Introduce the following conditions.

H1. The function $f(t, x, \tilde{x})$ is continuous in $\left(\tau_{i-1}, \tau_{i}\right] \times \mathbb{R}^{n} \times \mathbb{R}^{n}, i=1,2, \ldots$.

H2. The function $f(t, x, \tilde{x})$ is Lipschitz continuous with respect to its second and third arguments $x$ and $\tilde{x}$, uniformly on $t \in\left(t_{0}, \infty\right)$, with a constant $L>0$.

H3. $f(t, 0,0)=0$ for $t \in\left(t_{0}, \infty\right)$.

H4. The functions $I_{i}(x), i=1,2, \ldots$, are continuous in $\mathbb{R}^{n}$ and $I_{i}(0)=0$.

H5. $t_{0} \equiv \tau_{0}<\tau_{1}<\ldots<\tau_{i}<\tau_{i+1}<\ldots$.

H6. $\lim _{i \rightarrow \infty} \tau_{i}=\infty$.

H7. The function $g(t, x)$ is continuous in $\left[t_{0}-h, \infty\right) \times \mathbb{R}^{n}$.

H8. The set $M_{t}(n-m)$ is an $(n-m)$-manifold in $\mathbb{R}^{n}$.

H9. The function $F(t, u)$ is continuous and quasimonotone increasing in $\left(\tau_{i-1}, \tau_{i}\right] \times$ $\Omega, i=1,2, \ldots$.

H10. $F(t, 0)=0$ for $t \in\left(t_{0}, \infty\right)$. 
H11. The functions $\psi_{i}: \Omega \rightarrow \mathbb{R}^{k}, \psi_{i}(u)=u+B_{i}(u), i=1,2, \ldots$ are monotone increasing in $\Omega$.

H12. $B_{i}(0)=0, i=1,2, \ldots$.

H13. For any $i=1,2, \ldots$ and $w \in \Omega$ the limit

$$
\lim _{\substack{(t, u) \rightarrow(t, w) \\ t>x_{i}}} F(t, u)
$$

exists.

In the proofs of the main results we shall use the following lemmas.

LEMMA 1 (Bainov and Simeonov [2]). Let the following conditions hold.

1. Conditions $\mathrm{H} 9, \mathrm{H} 11$ and $\mathrm{H} 13$ are met.

2. The function $u^{+}: J^{+}\left(t_{0}, u_{0}\right) \rightarrow \mathbb{R}^{k}$ is the maximal solution of the problem (2) and $u^{+}\left(\tau_{i}+0\right) \in \Omega$ if $\tau_{i} \in J^{+}\left(t_{0}, u_{0}\right)$.

3. The function $w: J^{+}\left(t_{0}, u_{0}\right) \rightarrow \Omega$ is piecewise-continuous with points of discontinuity of the first kind $\tau_{i}, \tau_{i} \in J^{+}\left(t_{0}, u_{0}\right)$, at which it is continuous from the left and is such that

$$
\begin{aligned}
& w\left(\tau_{i}+0\right) \in \Omega, \quad \tau_{i} \in J^{+}\left(t_{0}, u_{0}\right), \\
& w\left(t_{0}+0\right) \leq u_{0}, \\
& D w(t) \leq F(t, w(t)) \quad \text { for } t \in J^{+}\left(t_{0}, u_{0}\right) \backslash\left\{\tau_{i}\right\}
\end{aligned}
$$

where $D w(t)$ is any of the Dini derivatives of the function $w(t)$,

$$
w\left(\tau_{i}+0\right) \leq \psi_{i}\left(w\left(\tau_{i}\right)\right), \quad \tau_{i} \in J^{+}\left(t_{0}, u_{0}\right) .
$$

Then $w(t) \leq u^{+}(t), t \in J^{+}\left(t_{0}, u_{0}\right)$.

LEMMA 2 (Bainov and Simeonov [2]). Let the following conditions hold.

1. Conditions $\mathrm{H} 9, \mathrm{H} 11$ and $\mathrm{H} 13$ are met.

2. The function $u^{-}: J^{+}\left(t_{0}, u_{0}\right) \rightarrow \mathbb{R}^{k}$ is the minimal solution of the problem (2) and $u^{-}\left(\tau_{i}+0\right) \in \Omega$ if $\tau_{i} \in J^{+}\left(t_{0}, u_{0}\right)$.

3. The function $w: J^{+}\left(t_{0}, u_{0}\right) \rightarrow \Omega$ is piecewise-continuous with points of discontinuity of the first kind $\tau_{i}, \tau_{i} \in J^{+}\left(t_{0}, u_{0}\right)$, at which it is continuous from the left and is such that

$$
\begin{aligned}
& w\left(\tau_{i}+0\right) \in \Omega, \quad \text { for } \tau_{i} \in J^{+}\left(t_{0}, u_{0}\right), \\
& w\left(t_{0}+0\right) \geq u_{0}, \\
& D w(t) \geq F(t, w(t)) \quad \text { for } t \in J^{+}\left(t_{0}, u_{0}\right), t \neq \tau_{i}, \\
& w\left(\tau_{i}+0\right) \geq \psi_{i}\left(w\left(\tau_{i}\right)\right), \quad \tau_{i} \in J^{+}\left(t_{0}, u_{0}\right) .
\end{aligned}
$$

Then $w(t) \geq u^{-}(t), t \in J^{+}\left(t_{0}, u_{0}\right)$. 
LEMMA 3. Let the following conditions hold.

1. Conditions $\mathrm{H} 9, \mathrm{H} 11$ and $\mathrm{H} 13$ are met.

2. The function $u^{+}: J^{+}\left(t_{0}, u_{0}\right) \rightarrow \mathbb{R}^{k}$ is the maximal solution of the problem (2) and $u^{+}\left(\tau_{i}+0\right) \in \Omega$ if $\tau_{i} \in J^{+}\left(t_{0}, u_{0}\right)$.

3. The functions $k:\left[t_{0}, \infty\right) \rightarrow(0, \infty)$ and $w: J^{+}\left(t_{0}, u_{0}\right) \rightarrow \Omega$ are piecewisecontinuous with points of discontinuity of the first kind $\tau_{i}, \tau_{i} \in J^{+}\left(t_{0}, u_{0}\right)$, at which they are continuous from the left and are such that

$$
\begin{aligned}
& k(t) w(t) \in \Omega \text { for } t \in J^{+}\left(t_{0}, u_{0}\right), \\
& k\left(\tau_{i}+0\right) w\left(\tau_{i}+0\right) \in \Omega \text { for } \tau_{i} \in J^{+}\left(t_{0}, u_{0}\right), \\
& k\left(t_{0}+0\right) w\left(t_{0}+0\right) \leq u_{0}, \\
& D k(t) w(t) \leq F(t, k(t) w(t)) \text { for } t \in J^{+}\left(t_{0}, u_{0}\right), t \neq \tau_{i}, \\
& k\left(\tau_{i}+0\right) w\left(\tau_{i}+0\right) \leq \psi_{i}\left(k\left(\tau_{i}\right) w\left(\tau_{i}\right)\right) \text { for } \tau_{i} \in J^{+}\left(t_{0}, u_{0}\right) .
\end{aligned}
$$

Then $k(t) w(t) \leq u^{+}(t)$ for $t \in J^{+}\left(t_{0}, u_{0}\right)$.

Lemma 3 is an immediate corollary of Lemma 1.

\section{Main results}

THEOREM 1. Let the following conditions hold.

1. Conditions $\mathrm{H} 1-\mathrm{H} 13$ are met.

2. The function $\gamma:\left[t_{0}, \infty\right) \rightarrow[1, \infty)$ is continuous in $\left[t_{0}, \infty\right)$.

3. There exist functions $V \in \mathscr{V}_{0}$ and $a, b \in \mathscr{K}$ such that

$$
\begin{aligned}
& a(|g(t, x)|) e \leq V(t, x) \leq \gamma(t) b(|g(t, x)|) e, \quad(t, x) \in\left[t_{0}, \infty\right) \times \mathbb{R}^{n}, \\
& \dot{V}_{(1)}(t, x) \leq F(t, V(t, x)), \quad(t, x) \in \cup_{i=1}^{\infty} G_{i}, \\
& V\left(\tau_{i}+0, x+I_{i}(x)\right) \leq \psi_{i}\left(V\left(\tau_{i}, x\right)\right), \quad i=1,2, \ldots, x \in \mathbb{R}^{n} .
\end{aligned}
$$

4. $J^{+}\left(t_{0}, u_{0}\right)=\left[t_{0}, \infty\right)$.

Then:

1. If the zero solution of the problem (2) is stable, then the zero solution of the problem (1) is stable with respect to the function $g(t, x)$.

2. If the zero solution of the problem (2) is globally equi-attractive, then the zero solution of the problem (1) is globally equi-attractive with respect to the function $g(t, x)$.

PROOF OF ASSERTION 1. Let $\varepsilon>0$ be chosen. From the stability of the zero solution of the problem (2) it follows that there exists a positive function $\delta^{*}=\delta^{*}\left(t_{0}, \varepsilon\right)$ which is continuous with respect to $t_{0} \in \mathbb{R}$ for each $\varepsilon>0$ and is such that if 
$0 \leq u_{0} \leq \delta^{*} e$ and $t \in J^{+}\left(t_{0}, u_{0}\right)$, then $u^{+}\left(t ; t_{0}, u_{0}\right)<a(\varepsilon) e$. By condition 4 of Theorem $1 J^{+}\left(t_{0}, u_{0}\right)=\left[t_{0}, \infty\right)$.

Let

$$
\delta=\delta\left(t_{0}, \varepsilon\right)=b^{-1}\left(\frac{\delta^{*}\left(t_{0}, \varepsilon\right)}{\gamma(t)}\right)
$$

and let $\varphi_{0} \in M_{t_{0}}(n-m)(\bar{\delta})$. It is clear that $\gamma\left(t_{0}\right) b\left(\left\|g\left(t, \varphi_{0}\right)\right\|\right) \leq \delta^{*}$. From (3) there follow the inequalities

$$
V\left(t_{0}+0, \varphi_{0}\left(t_{0}\right)\right) \leq \gamma\left(t_{0}\right) b\left(\left|g\left(t_{0}, \varphi_{0}\left(t_{0}\right)\right)\right|\right) e \leq \gamma\left(t_{0}\right) b\left(\left\|g\left(t, \varphi_{0}\right)\right\|\right) e \leq \delta^{*} e .
$$

Hence $u^{+}\left(t ; t_{0}, V\left(t_{0}+0, \varphi_{0}\left(t_{0}\right)\right)\right)<a(\varepsilon) e$ for $t \geq t_{0}$.

On the other hand, if $x(t)=x\left(t ; t_{0}, \varphi_{0}\right)$ is a solution of the problem (1), then from the conditions of Theorem 1 it follows that the function $w(t)=V(t, x(t))$ satisfies the conditions of Lemma 1. Using this fact and (3) as well, we obtain

$$
a(|g(t, x(t))|) e \leq V(t, x(t)) \leq u^{+}\left(t ; t_{0}, V\left(t_{0}+0, \varphi_{0}\left(t_{0}\right)\right)\right) \leq a(\varepsilon) e
$$

for $t \in J^{+}\left(t_{0}, \varphi_{0}\right)$. Hence $|g(t, x(t))|<\varepsilon$ for $t \in J^{+}\left(t_{0}, \varphi_{0}\right)$, that is, $x(t) \in M_{t}(n-$ $m)(\varepsilon)$ for $t \in J^{+}\left(t_{0}, \varphi_{0}\right)$.

PROOF OF ASSERTION 2. Let $\alpha>0, \varepsilon>0$. Introduce the notation

$$
\alpha^{*}=\gamma\left(t_{0}\right) b(\alpha)>0 \text {. }
$$

If the zero solution of the problem (2) is equi-attractive, then there exists a positive number $T=T\left(t_{0}, \alpha, \varepsilon\right)$ such that if $0 \leq u_{0} \leq \alpha^{*} e$, then $t_{0}+T \in J^{+}\left(t_{0}, u_{0}\right)$ and $u^{+}\left(t ; t_{0}, u_{0}\right)<a(\varepsilon) e$ for $t \in\left[t_{0}+T, \infty\right) \cap J^{+}\left(t_{0}, u_{0}\right)$. Since condition 4 of Theorem 1 is met, then $J^{+}\left(t_{0}, u_{0}\right)=\left[t_{0}, \infty\right)$.

Let $\varphi_{0} \in M_{t_{0}}(n-m)(\bar{\alpha})$. Then the inequality $\gamma\left(t_{0}\right) b\left(\left\|g\left(t, \varphi_{0}\right)\right\|\right) \leq \alpha^{*}$ is valid. From the inequalities (3) we obtain

$$
V\left(t_{0}+0, \varphi_{0}\left(t_{0}\right)\right) \leq \gamma\left(t_{0}\right) b\left(\left|g\left(t_{0}, \varphi_{0}\left(t_{0}\right)\right)\right|\right) e \leq \gamma\left(t_{0}\right) b\left(\left\|g\left(t, \varphi_{0}\right)\right\|\right) e \leq \alpha^{*} e .
$$

Hence $u^{+}\left(t ; t_{0}, V\left(t_{0}+0, \varphi_{0}\left(t_{0}\right)\right)\right)<a(\varepsilon) e$ for $t \geq t_{0}+T$.

If $x(t)=x\left(t ; t_{0}, \varphi_{0}\right)$ is the solution of the problem (1), then from Lemma 1 it follows that

$$
V(t, x(t)) \leq u^{+}\left(t ; t_{0}, V\left(t_{0}+0, \varphi_{0}\left(t_{0}\right)\right)\right) \quad \text { for } t \in J^{+}\left(t_{0}, \varphi_{0}\right) .
$$

From the above inequality and (3) we derive the inequalities

$$
a(|g(t, x(t))|) e \leq V(t, x(t)) \leq u^{+}\left(t ; t_{0}, V\left(t_{0}+0, \varphi_{0}\left(t_{0}\right)\right)\right) \leq a(\varepsilon) e
$$

for $t \in\left[t_{0}+T, \infty\right) \cap J^{+}\left(t_{0}, \varphi_{0}\right)$. Hence $|g(t, x(t))|<\varepsilon$ for $t \in\left[t_{0}+T, \infty\right) \cap J^{+}\left(t_{0}, \varphi_{0}\right)$. This completes the proof of Assertion 2 of Theorem 1. 
COROLLARY 1. Let the following conditions hold.

1. Conditions $\mathrm{H1}-\mathrm{H} 8$ are satisfied.

2. The functions $d:\left[t_{0}, \infty\right) \rightarrow \mathbb{B}_{+}$and $\gamma:\left[t_{0}, \infty\right) \rightarrow[1, \infty)$ are continuous in $\left[t_{0}, \infty\right)$.

3. There exist functions $V \in \mathscr{V}_{0}, a, b, c \in \mathscr{K}$ such that

$$
\begin{aligned}
& a(|g(t, x)|) \leq V(t, x) \leq \gamma(t) b(|g(t, x)|), \quad(t, x) \in\left[t_{0}, \infty\right) \times \mathbb{R}^{n}, \\
& \dot{V}_{(1)}(t, x) \leq d(t) c(|g(t, x)|), \quad(t, x) \in \cup_{i=1}^{\infty} G_{i}, \\
& V\left(\tau_{i}+0, x+I_{i}(x)\right) \leq V\left(\tau_{i}, x\right), \quad i=1,2, \ldots, x \in \mathbb{R}^{n} .
\end{aligned}
$$

4. $\int_{0}^{\infty} d(s) c\left[b^{-1}\left(\frac{\eta}{\gamma(s)}\right)\right] d s=\infty$ for sufficiently small $\eta>0$.

Then the zero solution of the problem (1) is globally equi-asymptotically stable with respect to the function $g(t, x)$.

Proof. Consider the scalar problem

$$
\begin{aligned}
\frac{d u}{d t} & =-d(t) c\left[b^{-1}\left(\frac{u}{\gamma(t)}\right)\right], \quad t>t_{0}, \\
u\left(t_{0}+0\right) & =u_{0}
\end{aligned}
$$

without impulses (that is, $B_{i}(u) \equiv 0$ ). By condition 4 of Corollary 1 the zero solution of the problem (6) is globally equi-asymptotically stable. Then from Theorem 1 it follows that the zero solution of the problem (1) is globally equi-asymptotically stable with respect to the function $g(t, x)$.

THEOREM 2. Let the following conditions hold.

1. Conditions $\mathrm{H} 1-\mathrm{H} 13$ are fulfilled.

2. There exist functions $V \in \mathscr{V}_{0}$, and $a, b \in \mathscr{K}$ such that

$$
\begin{aligned}
& a(|g(t, x)|) e \leq V(t, x) \leq b(|g(t, x)|) e \quad \text { for }(t, x) \in\left[t_{0}, \infty\right) \times \mathbb{R}^{n}, \\
& \dot{V}_{(1)}(t, x) \leq F(t, V(t, x)), \quad(t, x) \in \cup_{i=1}^{\infty} G_{i}, \\
& V\left(\tau_{i}+0, x+I_{i}(x)\right) \leq \psi_{i}\left(V\left(\tau_{i}, x\right)\right), \quad i=1,2, \ldots, x \in \mathbb{R}^{n} .
\end{aligned}
$$

3. $J^{+}\left(t_{0}, u_{0}\right)=\left[t_{0}, \infty\right)$.

Then:

1. If the zero solution of the problem (2) is uniformly stable, then the zero solution of the problem (1) is uniformly stable with respect to the function $g(t, x)$.

2. If the zero solution of the problem (2) is uniformly globally attractive, then the zero solution of the problem (1) is uniformly globally attractive with respect to the function $g(t, x)$. 
The proof of Theorem 2 is analogous to the proof of Theorem 1. We shall note that in this case we can choose the function $\delta^{*}$ (hence the function $\delta$ as well) and the number $T$ independently of $t_{0}$.

THEOREM 3. Let the conditions of Theorem 1 hold and $a(r) \rightarrow \infty$ as $r \rightarrow \infty$.

Then:

1. If the solutions of the problem (2) are equi-bounded, then the solutions of the problem (1) are equi-bounded with respect to the function $g(t, x)$.

2. If the solutions of the problem (2) are ultimately bounded for bound $N$, then the solutions of the problem (1) are ultimately bounded for bound $a^{-1}(N)$ with respect to the function $g(t, x)$.

Proof OF ASSERTION 1. Let $\alpha>0$. Set $\alpha^{*}=\gamma\left(t_{0}\right) b(\alpha)$. Then from the condition $a(r) \rightarrow \infty$ as $r \rightarrow \infty$ it follows that $\alpha \rightarrow \infty$ as $\alpha^{*} \rightarrow \infty$.

If the solutions of the problem (2) are equi-bounded, then there exists a positive function $\beta_{1}=\beta_{1}\left(t_{0}, \alpha\right)$ which is continuous with respect to $t_{0}$ for any $\alpha>0$ and is such that if $0 \leq u_{0} \leq \alpha^{*} e$ and $t \in J^{+}\left(t_{0}, u_{0}\right)$, then $u^{+}\left(t ; t_{0}, u_{0}\right)<\beta_{1} e$. By condition 4 of Theorem 1 we have $J^{+}\left(t_{0}, u_{0}\right)=\left[t_{0}, \infty\right)$. Set $\beta=\beta\left(t_{0}, \alpha\right)=a^{-1}\left(\beta_{1}\left(t_{0}, \alpha\right)\right)$. Let $\varphi_{0} \in M_{t_{0}}(n-m)(\bar{\alpha})$. Then $\gamma\left(t_{0}\right) b\left(\left\|g\left(t, \varphi_{0}\right)\right\|\right) \leq \alpha^{*}$ and since

$$
V\left(t_{0}+0, \varphi_{0}\left(t_{0}\right)\right) \leq \gamma\left(t_{0}\right) b\left(\left|g\left(t_{0}, \varphi_{0}\left(t_{0}\right)\right)\right|\right) e \leq \gamma\left(t_{0}\right) b\left(\left\|g\left(t, \varphi_{0}\right)\right\|\right) e,
$$

then $V\left(t_{0}+0, \varphi_{0}\left(t_{0}\right)\right) \leq \alpha^{*} e$. Hence $u^{+}\left(t ; t_{0}, V\left(t_{0}+0, \varphi_{0}\left(t_{0}\right)\right)\right)<\beta_{1} e$ for $t \geq t_{0}$. On the other hand, from Lemma 1 it follows that if $x(t)=x\left(t ; t_{0}, \varphi_{0}\right)$ is the solution of the problem (1), then $V(t, x(t)) \leq u^{+}\left(t ; t_{0}, V\left(t_{0}+0, \varphi_{0}\left(t_{0}\right)\right)\right)$ for $t \in J^{+}\left(t_{0}, \varphi_{0}\right)$.

From (3) we get to the inequalities

$$
a(|g(t, x(t))|) e \leq V(t, x(t)) \leq u^{+}\left(t ; t_{0}, V\left(t_{0}+0, \varphi_{0}\left(t_{0}\right)\right)\right) \leq \beta_{1} e
$$

for $t \in J^{+}\left(t_{0}, \varphi_{0}\right)$, whence it follows that $|g(t, x(t))|<a^{-1}\left(\beta_{1}\right)=\beta$ for $t \in$ $J^{+}\left(t_{0}, \varphi_{0}\right)$.

PROOF OF ASSERTION 2. Let $\alpha>0$ and $\alpha^{*}=\gamma\left(t_{0}\right) b(\alpha)$. If the solutions of the problem (2) are ultimately bounded for bound $N$, then there exist positive numbers $N$ and $T=T\left(t_{0}, \alpha\right)$ such that if $0 \leq u_{0} \leq \alpha e$ and $t \geq t_{0}+T$, then $u^{+}\left(t ; t_{0}, u_{0}\right)<N e$.

Let $\varphi_{0} \in M_{t_{0}}(n-m)(\bar{\alpha})$. Then $\gamma\left(t_{0}\right) b\left(\left\|g\left(t, \varphi_{0}\right)\right\|\right) \leq \alpha^{*}$. From condition (3) we derive the estimate $V\left(t_{0}+0, \varphi_{0}\left(t_{0}\right)\right)<\alpha^{*} e$. Consequently

$$
u^{+}\left(t ; t_{0}, V\left(t_{0}+0, \varphi_{0}\left(t_{0}\right)\right)\right)<N e \text { for } t \geq t_{0}+T \text {. }
$$

From Lemma 1 it follows that for $t \in J^{+}\left(t_{0}, \varphi_{0}\right)$ the inequality $V(t, x(t)) \leq$ $u^{+}\left(t ; t_{0}, V\left(t_{0}, \varphi_{0}\left(t_{0}\right)\right)\right)$ is valid, where $x(t)=x\left(t ; t_{0}, \varphi_{0}\right)$. Hence

$$
a(|g(t, x(t))|) e \leq V(t, x(t)) \leq u^{+}\left(t ; t_{0}, V\left(t_{0}+0, \varphi_{0}\left(t_{0}\right)\right)\right)<N e
$$


for $t \in\left[t_{0}+T, \infty\right) \cap J^{+}\left(t_{0}, \varphi_{0}\right)$ whence it follows that $|g(t, x(t))|<a^{-1}(N)$ for $t \in\left[t_{0}+T, \infty\right) \cap J^{+}\left(t_{0}, \varphi_{0}\right)$.

THEOREM 4. Let the conditions of Theorem 2 hold and $a(r) \rightarrow \infty$ as $r \rightarrow \infty$. Then:

1. If the solutions of the problem (2) are uniformly bounded, then the solutions of the problem (1) are uniformly bounded with respect to the function $g(t, x)$.

2. If the solutions of the problem (2) are uniformly ultimately bounded for bound $N$, then the solutions of the problem (1) are uniformly ultimately bounded for bound $a^{-1}(N)$ with respect to the function $g(t, x)$.

The proof of Theorem 4 is analogous to the proof of Theorem 3 . In this case the function $\beta$ and the number $T$ can be chosen independently of $t_{0}$.

THEOREM 5. Let the following conditions hold:

1. Conditions $\mathrm{H} 1-\mathrm{H} 13$ are valid.

2. There exists a function $k:\left[t_{0}, \infty\right) \rightarrow(0, \infty)$ which is piecewise-continuous with points of discontinuity of the first kind $\tau_{i}$ at which it is continuous from the left, $k(t) \rightarrow \infty$ as $t \rightarrow \infty$ and $k\left(\tau_{i}+0\right)>0$ for $i=1,2, \ldots$.

3. There exists a function $\gamma:\left[t_{0}, \infty\right) \rightarrow[1, \infty)$ which is continuous in $\left[t_{0}, \infty\right)$.

4. There exist functions $V \in \mathscr{V}_{0}, a, b \in \mathscr{K}$ such that

$$
\begin{aligned}
& a(|g(t, x)|) e \leq V(t, x) \leq \gamma(t) b(|g(t, x)|) e, \quad(t, x) \in\left[t_{0}, \infty\right) \times \mathbb{B}^{n}, \\
& D^{+} k(t) V(t, x) \leq F(t, k(t) V(t, x)), \quad(t, x) \in \cup_{i=1}^{\infty} G_{i},
\end{aligned}
$$

where $D^{+} k(t) V(t, x)=\underset{\sigma \rightarrow 0^{+}}{\limsup } \sigma^{-1}[k(t+\sigma) V(t+\sigma, x+\sigma f(t, x, \tilde{x}))-k(t) V(t, x)]$,

$$
k\left(\tau_{i}+0\right) V\left(\tau_{i}+0, x+I_{i}(x)\right) \leq \psi_{i}\left(k\left(\tau_{i}\right) V\left(\tau_{i}, x\right)\right), \quad i=1,2, \ldots, x \in \mathbb{R}^{n} .
$$

5. $J^{+}\left(t_{0}, \varphi_{0}\right)=\left[t_{0}, \infty\right)$.

Then, if the zero solution of the problem (2) is stable, then the zero solution of the problem (1) is globally equi-asymptotically stable with respect to the function $g(t, x)$.

PROOF. Let $\lambda=\inf _{\left.t \in i_{0}, \infty\right)} k(t)$. From condition 2 of Theorem 5 it follows that $\lambda>0$.

Let $\varepsilon>0$. If the zero solution of the problem (2) is stable, then there exists a positive function $\delta^{*}=\delta^{*}\left(t_{0}, \varepsilon\right)$ which is continuous with respect to $t_{0} \in \mathbb{R}$ for any $\varepsilon>0$ and is such that if $0 \leq u_{0} \leq \delta^{*} e$ and $t \geq t_{0}$, then $u^{+}\left(t ; t_{0}, u_{0}\right)<\lambda a(\varepsilon) e$.

Define $\delta>0$ as follows:

$$
\delta=\delta\left(t_{0}, \varepsilon\right)=b^{-1}\left(\frac{\delta^{*}\left(t_{0}, \varepsilon\right)}{\gamma\left(t_{0}\right) k\left(t_{0}\right)}\right) .
$$


Let $\varphi_{0} \in M_{t_{0}}(n-m)(\bar{\delta})$. Then $k\left(t_{0}\right) \gamma\left(t_{0}\right) b\left(\left\|g\left(t, \varphi_{0}\right)\right\|\right) \leq \delta^{*}$, whence by means of (7) we deduce the inequalities

$$
\begin{aligned}
k\left(t_{0}\right) V\left(t_{0}+0, \varphi_{0}\left(t_{0}\right)\right) & \leq k\left(t_{0}\right) \gamma\left(t_{0}\right) b\left(\left|g\left(t_{0}, \varphi_{0}\left(t_{0}\right)\right)\right|\right) e \\
& \leq k\left(t_{0}\right) \gamma\left(t_{0}\right) b\left(\left\|g\left(t, \varphi_{0}\right)\right\|\right) e \leq \delta^{*} e .
\end{aligned}
$$

Hence $u^{+}\left(t ; t_{0}, k\left(t_{0}\right) V\left(t_{0}+0, \varphi_{0}\left(t_{0}\right)\right)\right)<\lambda a(\varepsilon) e$ for $t \geq t_{0}$. From Lemma 3 there follows the validity of the inequality

$$
k(t) V(t, x(t)) \leq u^{+}\left(t ; t_{0}, k\left(t_{0}\right) V\left(t_{0}+0, \varphi_{0}\left(t_{0}\right)\right)\right)
$$

for $t \in J^{+}\left(t_{0}, \varphi_{0}\right)$, where $x(t)=x\left(t ; t_{0}, \varphi_{0}\right)$. Then for $t \in J^{+}\left(t_{0}, \varphi_{0}\right)$, the inequalities

$$
\lambda a(|g(t, x(t))|) e \leq k(t) V(t, x(t)) \leq u^{+}\left(t ; t_{0}, k\left(t_{0}\right) V\left(t_{0}+0, \varphi_{0}\left(t_{0}\right)\right)\right)<\lambda a(\varepsilon) e
$$

are valid from which it follows that $|g(t, x(t))|<\varepsilon$ for $t \in J^{+}\left(t_{0}, \varphi_{0}\right)$, that is, the zero solution of the problem (1) is stable.

We shall show that the zero solution of the problem (1) is globally equi-attractive. Let $\eta>0$. From the stability of the zero solution of the problem (2) it follows that there exists a positive function $\delta_{1}^{*}=\delta_{1}^{*}\left(t_{0}, \eta\right)$ which is continuous with respect to $t_{0} \in \mathbb{R}$ for any $\eta>0$ and is such that if $0 \leq u_{0} \leq \delta_{1}^{*} e$ and $t \geq t_{0}$, then $u^{+}\left(t ; t_{0}, u_{0}\right)<\eta e$.

Let $\alpha>0$. Choose $\eta>0$ so that

$$
\alpha=b^{-1}\left(\frac{\delta_{1}^{*}\left(t_{0}, \eta\right)}{k\left(t_{0}\right) \gamma\left(t_{0}\right)}\right)
$$

Let $\varphi_{0} \in M_{t_{0}}(n-m)(\bar{\alpha})$. Then

$$
k\left(t_{0}\right) \gamma\left(t_{0}\right) b\left(\left\|g\left(t, \varphi_{0}\right)\right\|\right) \leq \delta_{1}^{*},
$$

whence in view of (7) we deduce the inequalities

$$
\begin{aligned}
k\left(t_{0}\right) V\left(t_{0}+0, \varphi_{0}\left(t_{0}\right)\right) & \leq k\left(t_{0}\right) \gamma\left(t_{0}\right) b\left(\left|g\left(t_{0}, \varphi_{0}\left(t_{0}\right)\right)\right|\right) e \\
& \leq k\left(t_{0}\right) \gamma\left(t_{0}\right) b\left(\left\|g\left(t, \varphi_{0}\right)\right\|\right) e \leq \delta_{1}^{*} e .
\end{aligned}
$$

Hence $u^{+}\left(t ; t_{0}, k\left(t_{0}\right) V\left(t_{0}+0, \varphi_{0}\left(t_{0}\right)\right)\right)<\eta e$ for $t \geq t_{0}$. Lemma 3 implies for $t \in$ $J^{+}\left(t_{0}, \varphi_{0}\right)$ the validity of the inequality

$$
k(t) V(t, x(t)) \leq u^{+}\left(t ; t_{0}, k\left(t_{0}\right) V\left(t_{0}+0, \varphi_{0}\left(t_{0}\right)\right)\right),
$$

where $x(t)=x\left(t ; t_{0}, \varphi_{0}\right)$.

From the above inequality and (7) we get the inequalities

$$
\begin{aligned}
k(t) a(|g(t, x(t))|) e & \leq k(t) V(t, x(t)) \\
& \leq u^{+}\left(t ; t_{0}, k\left(t_{0}\right) V\left(t_{0}+0, \varphi_{0}\left(t_{0}\right)\right)\right)<\eta e,
\end{aligned}
$$


$t \in J^{+}\left(t_{0}, \varphi_{0}\right)$, which imply the estimate

$$
|g(t, x(t))|<a^{-1}\left(\frac{\eta}{k(t)}\right) \quad \text { for } t \in J^{+}\left(t_{0}, \varphi_{0}\right) .
$$

From condition 5 of Theorem 5 it follows that $J^{+}\left(t_{0}, \varphi_{0}\right)=\left[t_{0}, \infty\right)$.

Since $k(t) \rightarrow \infty$ as $t \rightarrow \infty$, then $a^{-1}(\eta / k(t)) \rightarrow 0$ as $t \rightarrow \infty$. Hence there exists a number $T^{*}=T^{*}\left(t_{0}, \alpha, \varepsilon\right)>t_{0}$ such that if $t \geq T^{*}$, then $|g(t, x(t))|<\varepsilon$. Let $T=T\left(t_{0}, \alpha, \varepsilon\right)=T^{*}-t_{0}$. Then for $t \geq t_{0}+T$ the inequality $|g(t, x(t))|<\varepsilon$ is valid, that is, the zero solution of the problem (1) is globally equi-attractive.

THEOREM 6. Let the conditions of Theorem 5 hold and $a(r) \rightarrow \infty$ as $r \rightarrow \infty$. Then, if the solutions of the problem (2) are equi-bounded, then the solutions of the problem (1) are equi-bounded and ultimately bounded for bound $N$ with respect to the function $g(t, x)$.

PROOF. Let $\lambda=\inf _{t \geq t_{0}} k(t)$. The condition $\lambda>0$ is valid.

We shall prove that if the solutions of the problem (2) are equi-bounded, then the solutions of the problem (1) are equi-bounded with respect to the function $g(t, x)$. Let $\alpha>0$. Set $\alpha^{*}=k\left(t_{0}\right) \gamma\left(t_{0}\right) b(\alpha)$. From the condition $a(r) \rightarrow \infty$ as $r \rightarrow \infty$ it follows that $\alpha \rightarrow \infty$ as $\alpha^{*} \rightarrow \infty$. If the solutions of the problem (2) are equi-bounded, then there exists a positive function $\beta_{1}=\beta_{1}\left(t_{0}, \alpha\right)$, continuous on $t_{0} \in \mathbb{R}$ for each $\alpha>0$ and such that if $0 \leq u_{0} \leq \alpha^{*} e$ and $t \geq t_{0}$, then $u^{+}\left(t ; t_{0}, u_{0}\right)<\lambda \beta_{1} e$. Set $\beta=\beta\left(t_{0}, \alpha\right)=a^{-1}\left(\beta_{1}\left(t_{0}, \alpha\right)\right)$.

Let $\varphi_{0} \in M_{t_{0}}(n-m)(\bar{\alpha})$. Then

$$
k\left(t_{0}\right) \gamma\left(t_{0}\right) b\left(\left\|g\left(t, \varphi_{0}\right)\right\|\right) \leq \alpha^{*}
$$

and since

$$
k\left(t_{0}\right) V\left(t_{0}+0, \varphi_{0}\left(t_{0}\right)\right) \leq k\left(t_{0}\right) \gamma\left(t_{0}\right) b\left(\left|g\left(t_{0}, \varphi_{0}\left(t_{0}\right)\right)\right|\right) e \leq k\left(t_{0}\right) \gamma\left(t_{0}\right) b\left(\left\|g\left(t, \varphi_{0}\right)\right\|\right) e,
$$

then

:

$$
k\left(t_{0}\right) V\left(t_{0}+0, \varphi_{0}\left(t_{0}\right)\right) \leq \alpha^{*} e .
$$

Hence $u^{+}\left(t ; t_{0}, k\left(t_{0}\right) V\left(t_{0}+0, \varphi_{0}\left(t_{0}\right)\right)\right)<\lambda \beta_{1} e$ for $t \geq t_{0}$.

From Lemma 3 and condition 5 of Theorem 5 there follows the validity of the inequality

$$
k(t) V(t, x(t)) \leq u^{+}\left(t ; t_{0}, k\left(t_{0}\right) V\left(t_{0}+0, \varphi_{0}\left(t_{0}\right)\right)\right) \quad \text { for } t \geq t_{0} .
$$

From the above inequality and (7) we deduce the inequalities

$$
\begin{aligned}
\lambda a(|g(t, x(t))|) e & \leq k(t) V(t, x(t)) \\
& \leq u^{+}\left(t ; t_{0}, k\left(t_{0}\right) V\left(t_{0}+0, \varphi_{0}\left(t_{0}\right)\right)\right)<\lambda \beta_{1} e, t \geq t_{0},
\end{aligned}
$$


which imply the validity of the estimate

$$
|g(t, x(t))|<a^{-1}\left(\beta_{1}\right)=\beta \quad \text { for } t \geq t_{0},
$$

that is, the solutions of the problem (1) are equi-bounded with respect to the function $g(t, x)$.

Let $\alpha>0$ and $\alpha^{*}=k\left(t_{0}\right) \gamma\left(t_{0}\right) b(\alpha)$. Choose a function $\beta^{*}=\beta^{*}\left(t_{0}, \alpha\right)$ so that if $0 \leq u_{0} \leq \alpha^{*} e$ and $t \geq t_{0}$, then $u^{+}\left(t ; t_{0}, u_{0}\right)<\beta^{*} e$. Let $\varphi_{0} \in M_{t_{0}}(n-$ $m)(\bar{\alpha})$. Then $k\left(t_{0}\right) \gamma\left(t_{0}\right) b\left(\left\|g\left(t, \varphi_{0}\right)\right\|\right) \leq \alpha^{*}$ and $k\left(t_{0}\right) V\left(t_{0}+0, \varphi_{0}\left(t_{0}\right)\right) \leq \alpha^{*} e$. Hence $u^{+}\left(t ; t_{0}, k\left(t_{0}\right) V\left(t_{0}+0, \varphi_{0}\left(t_{0}\right)\right)\right)<\beta^{*} e$ for $t \geq t_{0}$. From Lemma 3 and condition 5 of Theorem 5 we obtain that

$$
k(t) V(t, x(t)) \leq u^{+}\left(t ; t_{0}, k\left(t_{0}\right) V\left(t_{0}+0, \varphi_{0}\left(t_{0}\right)\right)\right) \quad \text { for } t \geq t_{0} .
$$

From the above inequality and (7) there follow the inequalities

$$
\begin{aligned}
k(t) a(|g(t, x(t))|) e & \leq k(t) V(t, x(t)) \leq \\
& \leq u^{+}\left(t ; t_{0}, k\left(t_{0}\right) V\left(t_{0}+0, \varphi_{0}\left(t_{0}\right)\right)\right) \leq \beta^{*} e, \quad t \geq t_{0},
\end{aligned}
$$

which imply the validity of the estimate

$$
|g(t, x(t))|<a^{-1}\left(\beta^{*} / k(t)\right), \quad t \geq t_{0} .
$$

From the condition $k(t) \rightarrow \infty$ as $t \rightarrow \infty$ it follows that $a^{-1}\left(\beta^{*} / k(t)\right) \rightarrow 0$ as $t \rightarrow \infty$. Consequently, if $N>0$, then there exists $T^{*}=T^{*}\left(t_{0}, \alpha\right)>t_{0}$ such that for $t \geq T^{*}$ the inequality $|g(t, x(t))|<N$ is valid. If $T=T\left(t_{0}, \alpha\right)=T^{*}-t_{0}>0$, then for $t \geq t_{0}+T$ we have $|g(t, x(t))|<N$, that is, the solutions of the problem (1) are ultimately bounded for bound $N$ with respect to the function $g(t, x)$.

THEOREM 7. Let the following conditions hold:

1. Conditions $\mathrm{H} 1-\mathrm{H} 13$ are satisfied.

2. For any $\delta>0$ and $t_{0} \in \mathbb{R}$ there exists a function $\varphi_{0} \in M_{t_{0}}(n-m)(\delta)$ such that $V\left(t_{0}+0, \varphi_{0}\left(t_{0}\right)\right)>0$.

3. There exist functions $V \in \mathscr{V}_{0}$, and $a, b \in \mathscr{K}$ such that

$$
\begin{aligned}
& V(t, x) \leq a(|g(t, x)|) e, \quad(t, x) \in\left[t_{0}, \infty\right) \times \mathbb{R}^{n}, \\
& \dot{V}_{(1)}(t, x) \geq F(t, V(t, x)), \quad(t, x) \in \cup_{i=1}^{\infty} G_{i}, \\
& V\left(\tau_{i}+0, x+l_{i}(x)\right) \geq \psi_{i}\left(V\left(\tau_{i}, x\right)\right), \quad i=1,2, \ldots, x \in \mathbb{R}^{n} .
\end{aligned}
$$

Then, if the zero solution of the problem (2) is unstable, then the zero solution of the problem (1) is unstable with respect to the function $g(t, x)$. 
PROOF. If the zero solution of the problem (2) is unstable, then there exist $\varepsilon^{*}>0$ and $t_{0} \in \mathbb{R}$ such that for any $\delta^{*}>0$ there exists $u_{0} \in \Omega: 0 \leq u_{0} \leq \delta^{*} e$ and $t^{*} \in J^{+}\left(t_{0}, u_{0}\right)$ for which we have

$$
u^{-}\left(t^{*} ; t_{0}, u_{0}\right) \nless \varepsilon e .
$$

Choose $\varepsilon>0$ such that $a(\varepsilon)<\varepsilon^{*}$. Let $\delta>0$. From condition 2 of Theorem 7 it follows that we can choose a function $\varphi_{0} \in M_{t_{0}}(n-m)(\delta)$ such that $V\left(t_{0}+0, \varphi_{0}\left(t_{0}\right)\right)>$ 0 . Let $\delta^{*}>0$ be chosen so that $0<\delta^{*} e \leq V\left(t_{0}+0, \varphi_{0}\left(t_{0}\right)\right)$. From Lemma 2 it follows that for any $t \in J^{+}\left(t_{0}, \varphi_{0}\right) \cap J^{+}\left(t_{0}, u_{0}\right)$ the inequality

$$
V(t, x(t)) \geq u^{-}\left(t ; t_{0}, u_{0}\right)
$$

is valid, where $x(t)=x\left(t ; t_{0}, \varphi_{0}\right)$.

We shall prove that there exists $t \in J^{+}\left(t_{0}, \varphi_{0}\right)$ for which $|g(t, x(t))| \geq \varepsilon$. Suppose that this is not true, that is, for any $t \in J^{+}\left(t_{0}, \varphi_{0}\right)$ the inequality

$$
|g(t, x(t))|<\varepsilon
$$

is valid. From inequalities (10), (14), (15), we get to

$$
\varepsilon^{*} e>a(\varepsilon) e>a\left(\left|g\left(t^{*}, x\left(t^{*}\right)\right)\right|\right) e \geq V\left(t^{*}, x\left(t^{*}\right)\right) \geq u^{-}\left(t^{*} ; t_{0}, u_{0}\right),
$$

which contradicts (13). Consequently, $|g(t, x(t))| \geq \varepsilon$ for some $t \in J^{+}\left(t_{0}, \varphi_{0}\right)$.

\section{Acknowledgement}

The present investigation was supported by the Bulgarian Ministry of Education, Science and Technologies under Grant MM-422.

\section{References}

[1] D. D. Bainov, V. C. Covachev and I. M. Stamova, "Stability under persistent disturbances of impulsive differential-difference equations of neutral type", J. Math. Anal. Appl., (to appear).

[2] D. D. Bainov and P. S. Simeonov, Systems with Impulse Effect: Stability, Theory and Applications Ellis Horwood Ltd., Chichester, (1989).

[3] P. Bhatia and V. Lakshmikantham, “An extension of Lyapunov's direct method", Mich. Math. J. 12 (1965) 183-191.

[4] G. K. Kulev and D. D. Bainov, "Lipschitz stability of impulsive systems of differential equations", Dynamics and Stability of Systems 8 (1993) 1-17.

[5] V. Lakshmikantham, D. D. Bainov and P. S. Simeonov, Theory of Impulsive Differential Equations World Scientific Publishers, Singapore, (1989). 
[6] V. D. Mil'man and A. D. Myshkis, "On the stability of motion in the presence of impulses", Siberian Math. J. 1 (1960) 233-237, (in Russian).

[7] P. S. Simeonov and D. D. Bainov, "Stability with respect to part of the variables in systems with impulse effect", J. Math. Anal. Appl. 117 (1986) 247-263.

[8] J. Vasundhara Devi, "A variation of the Lyapunov second method to impulsive differential equations”, J. Math. Anal. Appl. 117 (1993) 190-200. 